\title{
SYMPLECTIC BIEXTENSIONS AND A GENERALIZATION OF THE FOURIER-MUKAI TRANSFORM
}

\section{A. PolishchuK}

Let $A$ be an abelian variety over an algebraically closed field $k, \hat{A}$ be the dual abelian variety. The Fourier-Mukai transform is an equivalence between the derived categories of coherent sheaves $\mathcal{D}^{b}(A)$ and $\mathcal{D}^{b}(\hat{A})$. Notice that there is a "symplectic" line bundle $L_{A}$ on $(\hat{A} \times A)^{2}$, namely, $L_{A}=p_{14}^{*} \mathcal{P} \otimes p_{23}^{*} \mathcal{P}^{-1}$ where $\mathcal{P}$ is the Poincaré line bundle on $\hat{A} \times A$, such that the standard embeddings $A \subset \hat{A} \times A$ and $\hat{A} \subset \hat{A} \times A$ are "lagrangian" with respect to $L_{A}$, i.e. the restrictions of $L_{A}$ to $A^{2}$ and $\hat{A}^{2}$ are trivial (and they are maximal with this property). The purpose of this paper is to establish an analogous equivalence of derived categories for arbitrary lagrangian subvarieties in an abelian variety $X$ equipped with a line bundle $L$ over $X^{2}$ which satisfies some properties similar to that of $L_{A}$ ( $L$ should be a symplectic biextension - see below). Namely, with every lagrangian subvariety $Y \subset X$ we associate a canonical element of the Brauer group $e_{Y} \in \operatorname{Br}(X / Y)$ and consider the derived category $\mathcal{D}^{b}\left(X / Y, e_{Y}\right)$ of modules over the corresponding Azumaya algebra on $X / Y$. It turns out that for every pair of lagrangian subvarieties in $X$ there is an equivalence between these categories generalizing the Fourier-Mukai transform (more precisely, for this we have to assume that either char $k \neq 2$ or that there exists a biextension $P$ of $X^{2}$ such that $L \simeq P \otimes \sigma^{*} P^{-1}$ where $\sigma: X^{2} \rightarrow X^{2}$ is the permutation of factors). The class $e_{Y}$ is trivial if and only if the projection $X \rightarrow X / Y$ splits, in this case $\mathcal{D}^{b}\left(X / Y, e_{Y}\right) \simeq \mathcal{D}^{b}(X / Y)$. This implies the "if" part of the following conjecture: the derived categories of coherent sheaves on abelian varieties $A$ and $A^{\prime}$ are equivalent if and only if there is an isomorphism $f: \hat{A} \times A \widetilde{\rightarrow} \hat{A}^{\prime} \times A^{\prime}$ such that $(f \times f)^{*} L_{A^{\prime}} \simeq L_{A}$. In particular, for any abelian variety $A$ and a symmetric homomorphism $f: A \rightarrow \hat{A}$ we construct an equivalence $\mathcal{D}^{b}(A) \simeq \mathcal{D}^{b}\left(A / \operatorname{ker}\left(f_{n}\right)\right)$ where $f_{n}=\left.f\right|_{A_{n}}$ provided that $m n \operatorname{ker}(f)=0$ for some $m$ relatively prime to $n$.

The construction is based on analogy with the classical theory of representations of the Heisenberg group of a symplectic vector space: the categories $\mathcal{D}^{b}\left(X / Y, e_{Y}\right)$ are just different models of the same "irreducible" representation of the Heisenberg groupoid - a monoidal groupoid naturally

Received October 11, 1996. 
attached to $(X, L)$. The corresponding analogue of Weil representation is studied in [5].

\section{Symplectic biextensions}

Let $X$ be an abelian variety. A biextension of $X^{2}$ is a line bundle $L$ on $X^{2}$ together with isomorphisms

$$
\begin{aligned}
& L_{x+x^{\prime}, y} \simeq L_{x, y} \otimes L_{x^{\prime}, y}, \\
& L_{x, y+y^{\prime}} \simeq L_{x, y} \otimes L_{x, y^{\prime}}
\end{aligned}
$$

- this is a symbolic notation for isomorphisms $\left(p_{1}+p_{2}, p_{3}\right)^{*} L \simeq p_{13}^{*} L \otimes$ $p_{23}^{*} L$ and $\left(p_{1}, p_{2}+p_{3}\right)^{*} L \simeq p_{12}^{*} L \otimes p_{13}^{*} L$ on $X^{3}$, satisfying some natural cocycle conditions (see e.g. [1]).

A skew-symmetric biextension of $X^{2}$ is a biextension $L$ of $X^{2}$ together with an isomorphism of biextensions $\phi: \sigma^{*} L \widetilde{\longrightarrow} L^{-1}$, where $\sigma: X^{2} \rightarrow X^{2}$ is the permutation of factors, and a trivialization $\Delta^{*} L \simeq \mathcal{O}_{X}$ of $L$ over the diagonal $\Delta: X \rightarrow X^{2}$ compatible with $\phi$.

Every biextension $L$ of $X^{2}$ induces a homomorphism $\psi_{L}: X \rightarrow \hat{X}$ which is given on the level of points by $x \mapsto L_{x \times X}$. If $L$ is skew-symmetric, then $\widehat{\psi_{L}}=-\psi_{L}$. It is easy to see that $\psi_{L}=\psi_{L^{\prime}}$ if and only if $L$ and $L^{\prime}$ are isomorphic. A skew-symmetric homomorphism $\psi: X \rightarrow \hat{X}$ defines a skew-symmetric biextension by the formula $L(\psi)=(\psi \times \text { id })^{*} \mathcal{P}$, where $\mathcal{P}$ is the normalized Poincaré line bundle on $\hat{X} \times X$, provided that $\Delta^{*} L(\psi)=$ $(\psi, \mathrm{id})^{*} \mathcal{P}$ is trivial (apriori this is an element of 2 -torsion in $\left.\operatorname{Pic}(X)\right)$. In this case we have $\psi_{L(\psi)}=\psi$. A skew-symmetric biextension $L$ is called symplectic if $\psi_{L}$ is an isomorphism.

Let $Y \subset X$ be an abelian subvariety. Then $Y$ is called isotropic with respect to $L$ if there is an isomorphism of skew-symmetric biextensions $\left.L\right|_{Y \times Y} \simeq \mathcal{O}_{Y \times Y}$. This is equivalent to the condition that the composition

$$
Y \stackrel{i}{\rightarrow} X \stackrel{\psi_{L}}{\rightarrow} \hat{X} \stackrel{\hat{i}}{\rightarrow} \hat{Y}
$$

is zero. An isotropic subvariety $Y \subset X$ is called lagrangian if the morphism $Y \rightarrow \widehat{X / Y}$ induced by $\psi_{L}$ is an isomorphism. A skew-symmetric biextension $L$ of $X^{2}$ is called quasi-split if there exists a lagrangian subvariety in $X$. One can see easily that such a biextension is necessarily symplectic. The simplest example of an abelian variety with a symplectic biextension is $X=\hat{A} \times A$ for any abelian variety $A$ with the biextension $L_{A}=P \otimes \sigma^{*} P^{-1}$ where

$$
P=p_{14}^{*} \mathcal{P} \in \operatorname{Pic}(\hat{A} \times A \times \hat{A} \times A),
$$


$\mathcal{P}$ is the Poincaré line bundle on $A \times \hat{A}$. A symplectic biextension is called split if it is isomorphic to this one. Below we show how to construct all quasi-split symplectic biextensions.

Let $Y \subset X$ be a lagrangian subvariety. Let us denote $A=X / Y \simeq \hat{Y}$ so that there is an exact sequence

$$
0 \rightarrow \hat{A} \rightarrow X \stackrel{p}{\rightarrow} A \rightarrow 0
$$

such that $\left.\psi_{L}\right|_{\hat{A}}=\hat{p}$. The projection $p$ splits up to isogeny, that is there exists a homomorphism $s: A \rightarrow X$ such that $p s=n \operatorname{id}_{A}$.

Lemma 1.1. One can always choose a section $s: A \rightarrow X$ as above such that $\hat{s} \psi_{L} s=0$.

Proof. Start with any $s$ as above and then replace $n$ by $2 n^{2}$, and $s$ by $2 n s-\hat{s} \psi_{L} s$.

Choose $s: A \rightarrow X$ as in lemma, and let $\pi=($ id, $s): \hat{A} \times A \rightarrow X$ be the corresponding isogeny. Then since $\left.L\right|_{\hat{A}^{2}}$ and $(s \times s)^{*} L$ are trivial, it is easy to see that $\pi^{*} L \simeq L_{A}^{\otimes n}=p_{14}^{*} \mathcal{P}^{\otimes n} \otimes p_{23}^{*} \mathcal{P}^{\otimes-n}$. Thus, every quasisplit symplectic biextension descends from the power of the split one. It remains to determine which subgroups $\operatorname{ker}(\pi) \subset \hat{A} \times A$ can occur.

Let $P$ be any biextension of $X^{\prime} \times X^{\prime \prime}$. Then the restrictions of $P^{\otimes n}$ on $X_{n}^{\prime} \times X^{\prime \prime}$ and $X^{\prime} \times X_{n}^{\prime \prime}$ are canonically trivialized but these trivializations differ over $X_{n}^{\prime} \times X_{n}^{\prime \prime}$ by a bilinear morphism $e_{n}(P): X_{n}^{\prime} \times X_{n}^{\prime \prime} \rightarrow \mathbb{G}_{m}$. In the case of the Poincaré line bundle $\mathcal{P}$ over $\hat{A} \times A$ this construction gives a canonical perfect pairing $e_{n}: \hat{A}_{n} \times A_{n} \rightarrow \mathbb{G}_{m}$. In our situation the canonical trivializations of $L_{A}^{\otimes n}$ over $\left(\hat{A}_{n} \times A_{n}\right) \times(\hat{A} \times A)$ and $(\hat{A} \times$ $A) \times\left(\hat{A}_{n} \times A_{n}\right)$ differ over $\left(\hat{A}_{n} \times A_{n}\right)^{2}$ by a bilinear morphism $e_{n}\left(L_{A}\right)$ : $\left(\hat{A}_{n} \times A_{n}\right)^{2} \rightarrow \mathbb{G}_{m}$ which is given by the formula

$$
e_{n}\left(L_{A}\right)\left((\xi, x),\left(\xi^{\prime}, x^{\prime}\right)\right)=e_{n}\left(\xi, x^{\prime}\right) e_{n}\left(\xi^{\prime}, x\right),
$$

where $x, x^{\prime} \in A_{n}, \xi, \xi^{\prime} \in \hat{A}_{n}$.

By definition ker $\pi$ is the graph of a morphism $\phi: A_{n} \rightarrow \hat{A}_{n}$ induced by $s$. Now the biextension $L_{A}^{\otimes n}$ descends to $X$ if and only if there exist trivializations (as a biextension) of $L_{A}^{\otimes n}$ over $\operatorname{ker} \pi \times(\hat{A} \times A)$ and $(\hat{A} \times$ $A) \times \operatorname{ker} \pi$ which are compatible over $(\operatorname{ker} \pi)^{2}$. Since such trivializations are unique they coincide with the restrictions of the canonical trivializations above. Hence, the descent condition is that $\operatorname{ker}(\pi)$ is isotropic with respect to $e_{n}\left(L_{A}\right)$ which means that $\phi: A_{n} \rightarrow \hat{A}_{n}$ is skew-symmetric with respect to $e_{n}$, that is $\widehat{\phi}=-\phi$.

Thus, any quasi-split symplectic biextension arises from a pair $(A, \phi)$, where $\phi: A_{n} \rightarrow \hat{A}_{n}$ is a skew-symmetric morphism, as described above. It 
is easy to see that if we change $\phi$ by $\phi+f_{n}$ where $f_{n}$ is the restriction of a symmetric homomorphism $f: A \rightarrow \hat{A}$ to $A_{n}$ (then $f_{n}$ is automatically skew-symmetric), then we get isomorphic symplectic biextensions - this corresponds to a change of an isotropic morphism $s: A \rightarrow X$. Also, one can change $n$ by $n m$ and $\phi$ by the composition

$$
A_{n m} \stackrel{m}{\rightarrow} A_{n} \stackrel{\phi}{\rightarrow} \hat{A}_{n} \rightarrow \hat{A}_{n m}
$$

so that the corresponding symplectic biextension will be the same. However, this doesn't exhaust examples of pairs $(A, \phi)$ giving isomorphic biextensions. For example, it is easy to see that $A / \operatorname{ker}(\phi)=s(A) \subset X$ is a lagrangian subvariety in $X, X / s(A) \simeq \hat{A} / \phi\left(A_{n}\right)$, and the biextension associated with the pair $(A, \phi)$ corresponds also to the pair $\left(\hat{A} / \phi\left(A_{n}\right), \psi\right)$ where $\psi$ is the composition

$$
\psi:\left(\hat{A} / \phi\left(A_{n}\right)\right)_{n} \rightarrow \phi\left(A_{n}\right) \stackrel{\phi^{-1}}{\rightarrow} A_{n} / \operatorname{ker}(\phi) \rightarrow(A / \operatorname{ker}(\phi))_{n} .
$$

These considerations lead to the following theorem.

Theorem 1.2. Let $L$ be a symplectic biextension of $X^{2}$. For any lagrangian subvariety $Y \subset X$ there exists a lagrangian subvariety $Z \subset X$ such that $Y \cap Z$ is finite. Any pair of lagrangian subvarieties $(Y, Z)$ in $X$ such that $Y \cap Z$ is finite, is isomorphic to the pair $(\hat{A}, A / \operatorname{ker}(\phi))$ in $\hat{A} \times A /(\phi, \mathrm{id})\left(A_{n}\right)$ with its canonical symplectic biextension for some abelian variety $A$ and a skew-symmetric homomorphism $\phi: A_{n} \rightarrow \hat{A}_{n}$.

Proof. The first assertion is clear. To prove the second we should start with the lagrangian subspace $Y \subset X$ in the above argument and choose a splitting of $p: X \rightarrow X / Y$ up to isogeny which factors through $Z$. More precisely, let $f: Z \rightarrow X / Y$ be the restriction of $p$ to $Z$. Choose an isogeny $g: X / Y \rightarrow Z$ such that $f g=n \operatorname{id}_{X / Y}$. Then the composition of $g$ with the embedding of $Z$ in $X$ gives a lagrangian morphism $s: X / Y \rightarrow X$ such that $p s=n \operatorname{id}_{X / Y}$. Now we get an isogeny $\hat{A} \times A \rightarrow X$ as above (where $A=X / Y)$ such that $Y$ and $Z$ are the images of $\hat{A}$ and $A$ respectively, which finishes the proof.

Let us give an example of a quasi-split symplectic biextension which is not split. Let $A$ be a principally polarized abelian variety with $\operatorname{End}(A)=$ $\mathbb{Z}$. Then there is a symplectic isomorphism $\phi_{n}: A_{n} \rightarrow \hat{A}_{n}$ such that for every symmetric morphism $f: A \rightarrow \hat{A}$ the corresponding morphism $\left.f\right|_{A_{n}}$ is proportional to $\phi_{n}$. Now if $\operatorname{dim}(A)>1$ we can choose a symplectic morphism $\phi: A_{n} \rightarrow \hat{A}_{n}$ which is not proportional to $\phi_{n}$. It is easy to see that the corresponding symplectic biextension of $X^{2}$, where $X=\hat{A} \times$ $A /(\phi, \mathrm{id})\left(A_{n}\right)$, is not split. 


\section{Representations of the Heisenberg groupoid}

Let $X$ be an abelian variety and $L$ a symplectic biextension of $X^{2}$. Throughout this section we assume that there exists a biextension $P$ of $X^{2}$ such that $L \simeq P \otimes \sigma^{*} P^{-1}$ (an isomorphism of skew-symmetric biextensions). This is equivalent to the condition $\psi_{L}=f-\hat{f}$ for some $f: X \rightarrow \hat{X}$. For example, the quasi-split biextension associated with a pair $(A, \phi)$, where $\phi: A_{n} \rightarrow \hat{A}_{n}$ and $n$ is odd, satisfies this condition.

Definition 2.1. The Heisenberg groupoid $H(X)=H(X, P)$ is the stack of monoidal groupoids such that $H(X)(S)$ for a scheme $S$ over $k$ is the monoidal groupoid generated by the central subgroupoid $\mathcal{P} i c(S)$ of $\mathbb{G}_{m^{-}}$ torsors on $S$ and the symbols $T_{x}, x \in X(S)$ with the composition law

$$
T_{x} \circ T_{x^{\prime}}=P_{x, x^{\prime}} T_{x+x^{\prime}} .
$$

In other words, objects of $H(X)(S)$ are pairs $(M, x)$ where $M$ is a line bundle over $S, x \in X(S)$. A morphism $(M, x) \rightarrow\left(M^{\prime}, x^{\prime}\right)$ exists only if $x=x^{\prime}$ and is given by an isomorphism $M \rightarrow M^{\prime}$. The composition law is defined by the formula

$$
(M, x) \circ\left(M^{\prime}, x^{\prime}\right)=\left(P_{x, x^{\prime}} \otimes M \otimes M^{\prime}, x+x^{\prime}\right) .
$$

Denoting $T_{x}=\left(\mathcal{O}_{S}, x\right)$ we recover the above relation.

If we replace $P$ by $P^{\prime}=P \otimes \Lambda(M)$ for some line bundle $M$ on $X$ trivialized along the zero section, where $\Lambda(M)=\left(p_{1}+p_{2}\right)^{*} M \otimes p_{1}^{*} M^{-1} \otimes p_{2}^{*} M^{-1}$ (see e.g. [1]) we get an equivalent Heisenberg groupoid. The equivalence $H(X, P) \rightarrow H\left(X, P^{\prime}\right)$ is defined by the functor which is the identity on $\mathcal{P} i c(S)$ and sends $T_{x}$ to $M_{x}^{-1} T_{x}$. Since any symmetric biextension of $X^{2}$ has form $\Lambda(M)$ this shows that up to a non-unique equivalence the Heisenberg groupoid doesn't depend on a choice of $P$ such that $L=P \otimes \sigma^{*} P^{-1}$.

Remark. One can see easily that the Heisenberg groupoid can be considered as an extension of the group scheme $X$ by the stack of line bundles in the sense of Deligne (see [2]), namely, we associate to each point $x \in X(S)$ the trivial gerb of line bundles, and the composition is given by the formula above.

The Heisenberg groupoid $H(\hat{A} \times A)$ corresponding to a split biextension is generated by the Picard subgroupoid $\mathcal{P} i c$ and symbols $T_{x}, T_{y}$ where $x \in \hat{A}, y \in A$ with the following defining relations:

$$
\begin{aligned}
& T_{x} T_{x^{\prime}}=T_{x+x^{\prime}}, \\
& T_{y} T_{y^{\prime}}=T_{y+y^{\prime}}, \\
& T_{y} T_{x}=\langle x, y\rangle T_{x} T_{y} .
\end{aligned}
$$


Is is easy to see (see e.g. [5]) that the map $T_{y} \mapsto t_{y}^{*}, T_{x} \mapsto \cdot \otimes \mathcal{P}_{x}$ defines an action of $H(\hat{A} \times A)$ on $\mathcal{D}^{b}(A)$, where $t_{y}: A \rightarrow A$ is the translation by $y \in A, \mathcal{P}_{x}=\left.\mathcal{P}\right|_{x \times A}$ for $x \in \hat{A}$. Below we construct an analogous action for an arbitrary isotropic subvariety of an abelian variety with a symplectic biextension.

Let $Y \subset X$ be an isotropic subvariety. Then $\left.P\right|_{Y \times Y}$ has a natural structure of a symmetric biextension.

Definition 2.2. A pair $(Y, \alpha)$, where $Y$ is an isotropic abelian subscheme of $X$ with respect to $L$ and $\alpha$ is a line bundle on $Y$ with fixed trivialization along the zero section, is called isotropic if an isomorphism of symmetric biextensions of $Y \times Y$ is given:

$$
\left.\Lambda(\alpha) \simeq P\right|_{Y \times Y}
$$

which can be written symbolically as $P_{y, y^{\prime}}=\alpha_{y+y^{\prime}} \alpha_{y}^{-1} \alpha_{y^{\prime}}^{-1}$ for $y, y^{\prime} \in Y$.

For any isotropic subvariety $Y \subset X$ there exists $\alpha$ such that the pair $(Y, \alpha)$ is isotropic.

Definition 2.3. For an isotropic pair $(Y, \alpha)$ we define $\mathcal{F}(Y, \alpha)$ as the category of pairs $(\mathcal{A}, a)$ where $\mathcal{A} \in \mathcal{D}^{b}(X), a$ is an isomorphism in $\mathcal{D}^{b}(Y \times X)$ :

$$
a:\left.\left(i p_{1}+p_{2}\right)^{*} \mathcal{A} \widetilde{\rightarrow} P^{-1}\right|_{Y \times X} \otimes p_{1}^{*} \alpha^{-1} \otimes p_{2}^{*} \mathcal{A}
$$

where $i: Y \hookrightarrow X$ is the embedding, such that $(e \times \mathrm{id})^{*} a=\mathrm{id}$. This isomorphism can be written symbolically as follows:

$$
a_{y, x}: \mathcal{A}_{y+x} \stackrel{\widetilde{\rightarrow}}{\rightarrow} P_{y, x}^{-1} \alpha_{y}^{-1} \mathcal{A}_{x}
$$

where $y \in Y, x \in X$. These data should satisfy the following cocycle condition:

$$
\begin{aligned}
a_{y_{1}+y_{2}, x}= & a_{y_{2}, x} \circ a_{y_{1}, y_{2}+x}: \\
& \mathcal{A}_{y_{1}+y_{2}+x} \rightarrow P_{y_{1}, x+y_{2}}^{-1} P_{y_{2}, x}^{-1} \alpha_{y_{1}}^{-1} \alpha_{y_{2}}^{-1} \mathcal{A}_{x} \simeq P_{y_{1}+y_{2}, x}^{-1} \alpha_{y_{1}+y_{2}}^{-1} \mathcal{A}_{x},
\end{aligned}
$$

or in standard notation

$$
\left(p_{1}+p_{2}, p_{3}\right)^{*} a=\left(p_{2}, p_{3}\right)^{*} a \circ\left(p_{1}, i p_{2}+p_{3}\right)^{*} a
$$

in $\mathcal{D}^{b}(Y \times Y \times X)$. The morphisms between such pairs are morphisms between the corresponding objects in $\mathcal{D}^{b}(X)$ commuting with the isomorphisms in (2.1). 
It is easy to see that the category $\mathcal{F}(Y, \alpha)$ is equivalent to $\mathcal{D}^{b}(X / Y)$ provided the projection $p: X \rightarrow X / Y$ has a section $s: X / Y \rightarrow X$. However, in general this is not true: one encounters some twisted versions of $\mathcal{D}^{b}(X / Y)$ considered in the next section.

There is a natural action of the Heisenberg groupoid $H(X)$ on the category $\mathcal{F}(Y, \alpha)$ such that an object $(M, x)$ acts by the functor

$$
\left.\mathcal{A} \mapsto M \otimes P\right|_{X \times x} \otimes t_{x}^{*}(\mathcal{A}) .
$$

In the case $X=\hat{A} \times A, Y=\hat{A} \subset X$ this action coincides with the action of $H(\hat{A} \times A)$ on $\mathcal{D}^{b}(A) \simeq \mathcal{F}(\hat{A})$ mentioned above.

By analogy with the classical Heisenberg group it is natural to ask when these representations are irreducible in some sense. More precisely, for the construction of Weil representation it is relevant to know that all intertwining operators from Schrödinger representation to itself are proportional to the identity. As shown in [5] certain analogue of this property holds for the action of $H(\hat{A} \times A)$ on $\mathcal{D}^{b}(A)$. One can treat the case of an arbitrary lagrangian subvariety similarly, however, we don't need this result.

\section{Modules over Azumaya algebras}

We begin this section by briefly recalling the various ways to speak about the category of coherent modules over a scheme $S$ "twisted" by an element $e \in H^{2}\left(S, \mathbb{G}_{m}\right)$ : via Cech cocycles, gerbs, and Azumaya algebras. The simplest way to define such a category is to fix an open covering $\left(U_{i}\right)$ of $S$ (say, in flat topology) such that $e$ is represented by a Cech cocycle $\alpha_{i j k} \in \mathcal{O}^{*}\left(U_{i j k}\right)$ where $U_{i j k}=U_{i} \times{ }_{S} U_{j} \times{ }_{S} U_{k}$. Then we define $\operatorname{Coh}(S, \alpha)$ as the category of collections $\left(\mathcal{F}_{i}\right)$ of coherent sheaves on $U_{i}$ together with a system of isomorphisms $f_{i j}: \mathcal{F}_{i} \rightarrow \mathcal{F}_{j}$ over $U_{i j}=U_{i} \times_{S} U_{j}$ (such that $f_{j i}=f_{i j}^{-1}$ ) satisfying the twisted cocycle condition: $f_{j k} f_{i j}=\alpha_{i j k} f_{i k}$ over $U_{i j k}$. It is easy to see that up to equivalence this category depends only on the cohomology class of $\alpha$. The more abstract way to define this category (which doesn't involve a choice of covering) is to represent $e$ by a $\mathbb{G}_{m^{-}}$ gerb. Recall that a $\mathbb{G}_{m}$-gerb is a stack of groupoids $\mathcal{G}$ such that locally there is a unique isomorphism class of objects of $\mathcal{G}$ and the automorphism group of any object is $\mathbb{G}_{m}$. Equivalence classes of $\mathbb{G}_{m}$-gerbs over $S$ are in bijective correspondence with $H^{2}\left(S, \mathbb{G}_{m}\right)$. Now consider the category of representations of $\mathcal{G}$, i.e. the category of functors of stacks $\mathcal{G} \rightarrow \operatorname{Coh}(S)$ where $\operatorname{Coh}(S)$ is the stack of coherent sheaves. Choosing an open covering and a collection of objects $V_{i} \in \mathcal{G}\left(U_{i}\right)$ we arrive to the Cech description above. Sometimes $e$ is represented by a sheaf of Azumaya algebras $\mathcal{A}$ over $S$. Then locally $\mathcal{A}$ is isomorphic to a matrix algebra of rank $n^{2}$ over $S$. 
Now let $\mathcal{G}(\mathcal{A})$ be the $\mathbb{G}_{m}$-gerb of representations of $\mathcal{A}$ in locally free $\mathcal{O}_{S^{-}}$ modules of rank $n$. Then it is easy to see that $\mathcal{G}(\mathcal{A})$ represents the same cohomology class $e \in H^{2}\left(S, \mathbb{G}_{m}\right)$ and the categories of representations of $\mathcal{G}(\mathcal{A})$ and $\mathcal{A}$ in coherent sheaves on $S$ are equivalent. By abuse of notation we denote all these equivalent categories by $\operatorname{Coh}(S, e)$.

Let $E \rightarrow S$ be a $K$-torsor where $K$ is a finite flat commutative group scheme over $S$, let $0 \rightarrow \mathbb{G}_{m} \rightarrow G \rightarrow K \rightarrow 0$ be a central extension of $K$. Then it defines an element $e(G, E) \in H^{2}(S, e)$ such that the category of $G$-equivariant coherent sheaves on $E$ of weight 1 is equivalent to $\operatorname{Coh}(S, e(G, E))$. Here a weight of a $G$-equivariant coherent sheaf is defined as the weight of the induced $\mathbb{G}_{m}$-equivariant sheaf. Indeed, consider the gerb $\mathcal{G}(G, E)$ of liftings of $E$ to $G$-torsors (an object of $\mathcal{G}(G, E)$ over $U \rightarrow S$ is a $G$-torsor $\widetilde{E}$ over $U$ together with an isomorphism of $K$-torsors $\left.\widetilde{E} / \mathbb{G}_{m} \simeq E\right)$. Then we claim that the category of weight-1 $G$-equivariant sheaves is equivalent to the category of representations of $\mathcal{G}(G, E)^{o p}$ which is $\operatorname{Coh}(S, e)$ where $e$ is the inverse of the cohomology class of $\mathcal{G}(G, E)$. To see this note that a lifting of $E$ to a $G$-torsor can be considered as a weight-1 $G$-equivariant line bundle $L$ over $E$. A choice of such bundle over $E_{U}$ defines the equivalence $\mathcal{F} \mapsto \mathcal{F} \otimes L^{-1}$ of the category of weight-1 $G$-equivariant sheaves with the category of $K$-equivariant sheaves on $E_{U}$, therefore with $\operatorname{Coh}(U)$. This equivalence depends contravariantly on $L$, hence the assertion. The class $e(G, E)$ is trivial if and only if there is a global object of $\mathcal{G}(G, E)$, i.e. a global lifting of $E$ to a $G$-torsor. Also it is easy to see that $e(G, E)$ depends biadditively on the pair of classes $[G] \in H^{2}\left(K, \mathbb{G}_{m}\right),[E] \in H^{1}(S, K)$.

We apply this in the particular case when $S=A$ is an abelian variety, $p: E \rightarrow A$ is an isogeny of abelian varieties, so that $E$ can be considered as a $K$-torsor where $K=\operatorname{ker}(p)$. Then for any central extension $\pi: G \rightarrow K$ by $\mathbb{G}_{m}$ the previous construction gives a class $e(G, E) \in H^{2}\left(A, \mathbb{G}_{m}\right)$ which is an obstruction for existence of a line bundle $M$ over $E$ such that $K \subset$ $K(M)$ and $G$ is the restriction of Mumford's extension $G(M) \rightarrow K(M)$ to $K$ (see [4]). Let $\rho: G \rightarrow \mathrm{GL}_{n}$ be a weight-1 representation of $G$, $\bar{\rho}: K \rightarrow \mathrm{PGL}_{n}$ be the corresponding projective representation of $K$. Then the $\mathrm{PGL}_{n}$-torsor $E_{\bar{\rho}}$ on $A$ obtained as the push-forward of $E$ by $\bar{\rho}$ gives rise to an Azumaya algebra with the class $e(G, E)$. Consider $G$ as a $\mathbb{G}_{m}$-torsor over $K$ so that $G_{u}=\pi^{-1}(u)$ for $u \in K$. Let us denote by $\mathcal{O}_{K}(G)$ the corresponding line bundle over $K$. Then a weight-1 $G$-equivariant sheaf on $E$ can be described by the following data: a coherent sheaf $\mathcal{F}$ on $E$ and an isomorphism over $K \times E$ :

$$
p_{1}^{*} \mathcal{O}_{K}(G) \otimes p_{2}^{*} \mathcal{F} \rightarrow\left(i p_{1}+p_{2}\right)^{*} \mathcal{F}
$$


where $i: K \rightarrow E$ is the inclusion, satisfying the natural cocycle condition. The above construction gives an equivalence of this category with $\operatorname{Coh}(A, e(G, E))$.

We need also a derived category version of this equivalence. The slight difficulty is that derived categories of coherent sheaves don't glue well in any of standard topologies. However, as shown in the Appendix, the descent formalism for finite flat morphisms extends to derived categories. This allows to rephrase the definition of $\operatorname{Coh}(S, e)$ (e.g. in Cech version) for a class $e$ which is killed by a finite flat morphism $S^{\prime} \rightarrow S$ into a description of the corresponding derived category $\mathcal{D}^{b}(S, e)$. Similarly, one can describe the derived category of weight-1 $G$-equivariant sheaves above as the category of objects $\mathcal{F} \in \mathcal{D}^{b}(E)$ with isomorphisms (3.1) satisfying the cocycle condition and to show that it is equivalent to $\mathcal{D}^{b}(A, e(G, E))$.

Let $X$ be an abelian variety, $L=P \otimes \sigma^{*} P^{-1}$ be a symplectic biextension of $X^{2},(Y, \alpha)$ be an isotropic pair.

Proposition 3.1. There is a canonical class $e(Y) \in H^{2}\left(X / Y, \mathbb{G}_{m}\right)$ such that the category $\mathcal{F}(Y, \alpha)$ defined in the previous section is equivalent to $\mathcal{D}^{b}(X / Y, e(Y))$.

Proof. Choose a homomorphism of abelian varieties $s: Z \rightarrow X$ and a line bundle $\beta$ on $Z$ such that the composition $p s: Z \rightarrow X / Y$ is an isogeny and there is an isomorphism of biextensions of $s^{-1}(Y) \times Z$

$$
\left.\left.(s \times s)^{*} P\right|_{s^{-1}(Y) \times Z} \simeq \Lambda(\beta)\right|_{s^{-1}(Y) \times Z} .
$$

For example, one can take $Z=X / Y$ and $s^{\prime}: Z \rightarrow X$ such that $p s^{\prime}=$ $n \operatorname{id}_{X / Y}$, then $s^{\prime-1}(Y)=(X / Y)_{n}$ and $\left.\left(n s^{\prime} \times n s^{\prime}\right)^{*} P\right|_{(X / Y)_{n 2} \times X / Y}$ is a trivial biextension (see [1], 4.2), hence we can take $s=n s^{\prime}$ and $\beta=\mathcal{O}_{Z}$.

Then $\Lambda\left(\left.\left(\left.s\right|_{s^{-1}(Y)}\right)^{*} \alpha \otimes \beta^{-1}\right|_{s^{-1}(Y)}\right)$ is a trivial biextension, hence the $\mathbb{G}_{m^{-}}$ torsor $\left.\beta\right|_{s^{-1}(Y)} \otimes\left(\left.s\right|_{s^{-1}(Y)}\right)^{*} \alpha^{-1}$ defines a central extension $G$ of $s^{-1}(Y)$ by $\mathbb{G}_{m}$. It is easy to see that the class $e(G, Z) \in H^{2}\left(X / Y, \mathbb{G}_{m}\right)$ defined above doesn't depend on a choice of $\alpha$ such that the pair $(Y, \alpha)$ is isotropic. We claim also that it doesn't depend on a choice of $Z$ and $\beta$. Indeed, if we change $\beta$ by $\beta^{\prime}=\beta \otimes \gamma$ where $\left.\Lambda(\gamma)\right|_{s^{-1}(Y) \times Z}$ is trivial, then the new central extension is the sum of $G$ and the restriction of Mumford's extension $G(\gamma) \rightarrow K(\gamma)$ to $s^{-1}(Y)$. But $\gamma$ has a natural structure of $G(\gamma)$ equivariant line bundle, hence $e(G(\gamma), Z)=0$. Also, it is easy to see that $e(G, Z)$ doesn't change if we replace $s: Z \rightarrow X$ by the composition of $s$ with an isogeny $Z^{\prime} \rightarrow Z$. It remains to check that $e(G, Z)$ is invariant under the change $s^{\prime}=s+f$ where $f: Z \rightarrow Y$ is any homomorphism. In this case $s^{\prime-1}(Y)=s^{-1}(Y)$ and

$$
\left.\left.\left(s^{\prime} \times s^{\prime}\right)^{*} P\right|_{s^{-1}(Y) \times Z} \simeq \Lambda\left(\beta^{\prime}\right)\right|_{s^{-1}(Y) \times Z}
$$


where $\beta^{\prime}=\beta \otimes f^{*} \alpha \otimes(f, s)^{*} P$. On the other hand

$$
\left.\left(\left.s^{\prime}\right|_{s^{-1}(Y)}\right)^{*} \alpha \simeq\left(\left.s\right|_{s^{-1}(Y)}\right)^{*} \alpha \otimes\left(f^{*} \alpha \otimes(f, s)^{*} P\right)\right|_{s^{-1}(Y)}
$$

so that the corresponding central extension of $s^{-1}(Y)$ is the same.

Given an object $(\mathcal{A}, a)$ of $\mathcal{F}(Y, \alpha)$ where $\mathcal{A} \in \mathcal{D}^{b}(X)$, a is an isomorphism (2.1), we can consider $s^{*} \mathcal{A} \in \mathcal{D}^{b}(Z)$. Then $a$ induces an isomorphism

$$
a_{s(u), s(z)}: s^{*} \mathcal{A}_{u+z} \widetilde{\rightarrow}(s \times s)^{*} P_{u, z}^{-1} \alpha_{s(u)}^{-1} s^{*} \mathcal{A}_{z}
$$

where $u \in s^{-1}(Y), z \in Z$, satisfying the usual cocycle condition. Let $F(\mathcal{A})=s^{*} \mathcal{A} \otimes \beta$, then $a_{s(u), s(z)}$ together with (3.2) gives an isomorphism

$$
F(a): F(\mathcal{A})_{u+z} \widetilde{\rightarrow} \beta_{u} \otimes \alpha_{s(u)}^{-1} \otimes F(\mathcal{A})_{z}
$$

where $u \in s^{-1}(Y), z \in Z$. In other words, $(F(\mathcal{A}), F(a))$ can be considered as a weight-1 $G$-equivariant object of $\mathcal{D}^{b}(Z)$. This gives the required equivalence as one can check applying Theorem A of Appendix to the morphism $Y \times Z \rightarrow X$ and the trivial descent for the projection $Y \times Z \rightarrow Z$.

Remark. If $(Y, \alpha)$ and $(Z, \beta)$ are isotropic pairs, then $\mathbb{G}_{m}$-torsor $\left.\beta\right|_{Y \cap Z} \otimes$ $\left.\alpha\right|_{Y \cap Z} ^{-1}$ defines a central extension $G$ of $Y \cap Z$ by $\mathbb{G}_{m}$, which in case when $Y$ and $Z$ are lagrangian and $Y \cap Z$ is finite gives the class $e_{Y} \in H^{2}\left(X / Y, \mathbb{G}_{m}\right)$. The corresponding commutator form on $Y \cap Z$ measures the difference between the symmetric structures on $\left.P\right|_{(Y \cap Z)^{2}}$ restricted from $Y^{2}$ and $Z^{2}$. In other words, this is the standard skew-symmetric form associated with the biextension $\left.L\right|_{Y \times Z}$ measuring the difference between two trivializations of $\left.L\right|_{(Y \cap Z)^{2}}$ restricted from $Y \times(Y \cap Z)$ and $(Y \cap Z) \times Z$. When $Y$ and $Z$ are lagrangian and $Y \cap Z$ is finite, this form is non-degenerate since it corresponds to the canonical duality between $Y \cap Z=\operatorname{ker}(Y \rightarrow X / Z)=$ $\operatorname{ker}(Y \rightarrow \hat{Z})$ and $\operatorname{ker}(Z \rightarrow \hat{Y})=\operatorname{ker}(Z \rightarrow X / Y)=Y \cap Z$.

By definition the class $e(Y)$ vanishes if the projection $X \rightarrow X / Y$ splits. It turns out that if $Y$ is lagrangian then the converse is also true.

Proposition 3.2. Let $Y \subset X$ be a lagrangian subvariety. If $e_{Y}=0$ then the projection $X \rightarrow X / Y$ splits.

Proof. According to Theorem 1.2 we can assume $X=\hat{A} \times A /(\phi, \mathrm{id})\left(A_{n}\right)$, $Y=A / \operatorname{ker}(\phi) \subset X$ for an abelian variety $A$ and a skew-symmetric homomorphism $\phi: A_{n} \rightarrow \hat{A}_{n}$. Now we can take $Z=\hat{A} \subset X$ in the definition of $e_{Y}$. The kernel of the projection $Z \rightarrow X / Y$ is $\phi\left(A_{n}\right) \subset \hat{A}$ and the commutator form of its central extension considered above is (up to sign)

$$
e(\phi(x), \phi(y))=e_{n}(\phi(x), y)
$$


where $x, y \in A_{n}$. The triviality of $e_{Y}$ implies that there exists a symmetric homomorphism $g: \hat{A} \rightarrow A$ such that $\phi\left(A_{n}\right) \subset \operatorname{ker}(g)$ and $e=\left.e^{g}\right|_{\phi\left(A_{n}\right)^{2}}$ where $e^{g}$ is the standard symplectic form on $\operatorname{ker}(g)$. In other words, the following equality holds:

$$
e_{n}(\phi(x), y)=e_{n}\left(\phi(x), g\left(n^{-1} \phi(y)\right)\right)
$$

for all $x, y \in A_{n}$, which implies that $y-g\left(n^{-1} \phi(y)\right) \in \operatorname{ker}(\phi)$ for $y \in A_{n}$. Note that $x \mapsto g\left(n^{-1} x\right) \bmod (\operatorname{ker}(\phi))$ is a well-defined homomorphism $\hat{A} \rightarrow$ $A / \operatorname{ker}(\phi)$ since $g\left(A_{n}\right) \subset \operatorname{ker}(\phi)$ (which is obtained from $\phi\left(A_{n}\right) \subset \operatorname{ker}(g)$ by duality). Thus, the homomorphism

$$
\hat{A} \times A \rightarrow A / \operatorname{ker}(\phi):(x, y) \mapsto y-g\left(n^{-1} x\right) \bmod (\operatorname{ker}(\phi))
$$

descends to a homomorphism $X=\hat{A} \times A /(\phi$, id $)\left(A_{n}\right) \rightarrow A / \operatorname{ker}(\phi)=Y$ splitting the embedding $Y \rightarrow X$.

\section{Intertwining functors}

Let $X$ be an abelian variety with a symplectic biextension $L$ of $X^{2}$. In this section we construct an equivalence of $H(X)$-representations $\mathcal{F}(Y, \alpha) \simeq \mathcal{F}(Z, \beta)$ for isotropic pairs $(Y, \alpha)$ and $(Z, \beta)$ such that $Y$ and $Z$ are lagrangian.

The idea is to mimic the classical construction. Namely, consider the functor of "integration over $Z$ "

$$
R: \mathcal{F}(Y, \alpha) \rightarrow \mathcal{F}(Z, \beta): \mathcal{A} \mapsto p_{2 *}\left(\left.P\right|_{Z \times X} \otimes p_{1}^{*} \beta \otimes\left(i p_{1}+p_{2}\right)^{*} \mathcal{A}\right) .
$$

The following symbolic notation stresses the analogy with the classical case:

$$
R(\mathcal{A})_{x}=\int_{Z} P_{z, x} \beta_{z} \mathcal{A}_{z+x} d z .
$$

It easy to check that $R(\mathcal{A})$ has a natural structure of an object of $\mathcal{F}(Z, \beta)$ :

$$
\begin{aligned}
& R(\mathcal{A})_{z^{\prime}+x}=\int_{Z} P_{z, z^{\prime}+x} \beta_{z} \mathcal{A}_{z+z^{\prime}+x} d z \simeq \int_{Z} P_{z, x} \beta_{z+z^{\prime}} \beta_{z^{\prime}}^{-1} \mathcal{A}_{z+z^{\prime}+x} d z \simeq \\
& \int_{Z} P_{z-z^{\prime}, x} \beta_{z} \beta_{z^{\prime}}^{-1} \mathcal{A}_{z+x} d z \simeq P_{z^{\prime}, x}^{-1} \beta_{z^{\prime}}^{-1} R(\mathcal{A})_{x}
\end{aligned}
$$

- here we used the isomorphism $\left.P\right|_{Z^{2}} \simeq \Lambda(\beta)$ and the change of variable $z \mapsto z-z^{\prime}$. It is also clear that $R$ commutes with the action of $H(X)$. In the classical theory in order to get an invertible intertwining operator one should replace the integration over $Z$ by the integration over $Z / Y \cap Z$ in the above formula. This doesn't work literally in our context- it turns out that in the correct definition one eliminates the "excess" integration over the connected component of $Y \cap Z$, and over a "largangian half" of 
the group of connected components of $Y \cap Z$. Instead of working out the case when $\operatorname{dim}(Y \cap Z)>0$ we use the following simple lemma which allows to avoid it.

Lemma 4.1. For any pair $Y$ and $Z$ of lagrangian subvarieties of $X$ there exists a lagrangian subvariety $T \subset X$ such that the intersections $Y \cap T$ and $Z \cap T$ are finite.

Proof. We can work in the category of abelian varieties up to isogeny. We have an isogeny $X \sim Y \times \hat{Y}$ and $Z / Y \cap Z \subset Y \times \hat{Y}$ is isogenic to the graph of a symmetric morphism

$$
g: Z / Y \cap Z \rightarrow Y / Y \cap Z \sim Z \widehat{/ Y \cap Z} .
$$

Let $K \sim \operatorname{ker}(g)$. We have a decomposition $Z / Y \cap Z \sim K \times K^{\prime}$ such that $g$ is given by a symmetric isogeny $K^{\prime} \rightarrow \hat{K}^{\prime}$. Now let $\hat{Y} \sim Z / Y \cap Z \times K^{\prime \prime} \sim$ $K \times K^{\prime} \times K^{\prime \prime}$. Let us define a symmetric morphism $f: \hat{Y} \rightarrow Y$ to be a symmetric isogeny on $K$ and zero on two other factors. Then we can take the graph of $f$ to be $T$.

Thus, we may assume that $Y \cap Z$ is finite. We have a natural central extension $G$ of $Y \cap Z$ by $\mathbb{G}_{m}$ given by the $\mathbb{G}_{m}$-torsor $\left.\left.\beta\right|_{Y \cap Z} \otimes \alpha^{-1}\right|_{Y \cap Z}$ such that $\mathcal{F}(Y, \alpha)$ is equivalent to the category of weight- $1 G$-equivariant objects of $\mathcal{D}^{b}(Z)$, while $\mathcal{F}(Z, \beta)$ - to that of weight-1 $G^{-1}$-equivariant objects of $\mathcal{D}^{b}(Y)$, where $G^{-1}$ is the inverse central extension of $Y \cap Z$ (given by the inverse $\mathbb{G}_{m}$-torsor). Let $e$ be the commutator form of $G$. Then as we observed above $e$ is non-degenerate (i.e. $G$ is a non-degenerate theta-group in the terminology of [3]). Hence, we can choose a lagrangian subgroup $H \subset Y \cap Z$ and a trivialization of the central extension $G$ over $H$, which is the same as a lifting of $H$ to a subgroup in $G$ (cf. [3], Lemma 2.5.4). Then we can define the reduced functor

$$
\bar{R}: \mathcal{F}(Y, \alpha) \rightarrow \mathcal{F}(Z, \beta): \bar{R}(\mathcal{A})_{x}=\int_{Z / H} P_{z, x} \beta_{z} \mathcal{A}_{z+x} d z
$$

To give a meaning to this notice that an object $P_{z, x} \beta_{z} \mathcal{A}_{z+x} \in \mathcal{D}^{b}(Z \times X)$ descends canonically to an object of $\mathcal{D}^{b}(Z / H \times X)$ (use the additional data on $\mathcal{A} \in \mathcal{F}(Y, \alpha)$ and the isomorphism $\left.\left.\left.\alpha\right|_{H} \simeq \beta\right|_{H}\right)$. As above it is easy to check that $\bar{R}(\mathcal{A})$ has a natural structure of an object of $\mathcal{F}(Z, \beta)$ and $\bar{R}$ commutes with $H(X)$-action.

Theorem 4.2. The functor $\bar{R}$ is an equivalence of categories.

Proof. First let us rewrite $\bar{R}$ as the functor from the category of weight-1 $G$ equivariant objects of $\mathcal{D}^{b}(Z)$ to that of weight- $1 G^{-1}$-equivariant objects of $\mathcal{D}^{b}(Y)$ using the equivalences defined above. Recall that the equivalence of 
the first category with $\mathcal{F}(Y, \alpha)$ is given by the functor $F_{Y}$ which associates to $\mathcal{A} \in \mathcal{F}(Y, \alpha)$ the $G$-equivariant object $\left.\mathcal{A}\right|_{Y} \otimes \beta \in \mathcal{D}^{b}(Z)$, while the second equivalence is induced by $F_{Z}: \mathcal{F}(Z, \beta) \rightarrow \mathcal{D}^{b}(Y):\left.\mathcal{A}^{\prime} \mapsto \mathcal{A}^{\prime}\right|_{Y} \otimes \alpha$. Now for $\mathcal{A} \in \mathcal{F}(Y, \alpha)$ we have

$$
\begin{aligned}
F_{Z}(\bar{R}(\mathcal{A}))_{y} & =\alpha_{y} \int_{Z / H} P_{z, y} \beta_{z} \mathcal{A}_{z+y} d z \simeq \alpha_{y} \int_{Z / H} P_{z, y} \beta_{z} P_{y, z}^{-1} \alpha_{y}^{-1} \mathcal{A}_{z} d z \\
& \simeq \int_{Z / H} L_{z, y} F_{Y}(\mathcal{A})_{z} d z
\end{aligned}
$$

The latter integral should be understood in the same sense as above: the $G$-equivariance data on $F_{Y}(\mathcal{A})$ allow to descend $L_{z, y} F_{Y}(\mathcal{A})_{z}$ to an object of $\mathcal{D}^{b}(Z / H \times Y)$. Notice that $G$-equivariance data on an object $\mathcal{G} \in \mathcal{D}^{b}(Z)$ includes the descent data for the projection $Z \rightarrow Z / H$, so that $G$-equivariant objects of $\mathcal{D}^{b}(Z)$ can be considered as objects of $\mathcal{D}^{b}(Z / H)$ with some additional data. More precisely, the isomorphism

$$
\mathcal{G}_{z+u} \simeq G_{u} L_{z, u} \mathcal{G}_{z}
$$

where $u \in Y \cap Z$, induced by the $G$-equivariance data and the trivialization of $L_{z, u}$ commutes with the descent data for $Z \rightarrow Z / H$, so it induces an isomorphism of descended objects on $Z / H \times(Y \cap Z)$

$$
\overline{\mathcal{G}}_{z+u} \simeq G_{u} \bar{L}_{z, u} \overline{\mathcal{G}}_{z}
$$

— these are the additional data for $\overline{\mathcal{G}} \in \mathcal{D}^{b}(Z)$. Similar, we can consider $G^{-1}$-equivariant objects of $\mathcal{D}^{b}(Y)$ as objects of $\mathcal{D}^{b}(Y / H)$ with additional data. It is easy to see that biextension $L_{z, y}$ of $Z \times Y$ descends to a biextension $\bar{L}$ of $Z / H \times Y / H$ which induces an isomorphism $Z / H \cong \widehat{Y / H}$. Thus, $\bar{R}$ is compatible with the Fourier-Mukai transform $\mathcal{D}^{b}(Z / H) \rightarrow \mathcal{D}^{b}(Y / H)$ via the "forgetting" functors $\mathcal{F}(Y, \alpha) \rightarrow \mathcal{D}^{b}(Z / H)$ and $\mathcal{F}(Z, \beta) \rightarrow \mathcal{D}^{b}(Y / H)$ described above. Let $\bar{Q}: \mathcal{F}(Z, \beta) \rightarrow \mathcal{F}(Y, \alpha)$ be the functor defined in the same way as $\bar{R}$ but with $Y$ and $Z$ interchanged. Then it is compatible with the "inverse" Fourier transform $\mathcal{D}^{b}(Y / H) \rightarrow \mathcal{D}^{b}(Z / H)$ given by the kernel $\bar{L}_{y, z} \simeq \bar{L}_{z, y}^{-1}$. Its composition with the direct Fourier transform is isomorphic to a shift in the derived category and it is easy to see that this isomorphism extends to our additional data, so that $\bar{Q}$ is quasi-inverse to $\bar{R}$ up to shift.

Consider the following example. Let $X=\hat{A} \times A, L=L_{A}$ be the standard split symplectic biextension, $Y=\hat{A} \subset \hat{A} \times A$ be its standard lagrangian subvariety. Let $f: A \rightarrow \hat{A}$ be a symmetric morphism, $Z=$ $A / \operatorname{ker}\left(f_{n}\right) \simeq\left(f, n \operatorname{id}_{A}\right)(A) \subset \hat{A} \times A$ where $f_{n}=\left.f\right|_{A_{n}}$. Then it is easy to see that $Z$ is lagrangian. Assume in addition that $m n \operatorname{ker}(f)=0$ for some 
$m$ relatively prime to $n$. Then we claim that the projection $X \rightarrow X / Z$ splits. Indeed, changing $m$ if necessary we may assume that $m+k n=1$ for some integer $k$. Notice that we have an isomorphism $X / Z \simeq \hat{A} / f\left(A_{n}\right) \simeq$ $A /(n \operatorname{ker}(f))$. Now we can define the splitting morphism

$$
X / Z \simeq A /(n \operatorname{ker}(f)) \stackrel{(k, m)}{\longrightarrow} A / \operatorname{ker}(f) \times A \simeq X .
$$

Hence, $e_{Y}=e_{Z}=0$ and we get an equivalence of derived categories

$$
\mathcal{D}^{b}(A)=\mathcal{D}^{b}(X / Y) \simeq \mathcal{D}^{b}(X / Z) \simeq \mathcal{D}^{b}(Z)=\mathcal{D}^{b}\left(A / k e r\left(f_{n}\right)\right)
$$

- here we used the Fourier-Mukai equivalence for $Z$ and $X / Z \simeq \hat{Z}$.

The proof of Theorem 4.2 shows that we can eliminate the assumption that there exists a biextension $P$ of $X^{2}$ such that $L \simeq P \otimes \sigma^{*} P^{-1}$ once we can define the categories in question without it. In fact, if the characteristic of the base field is not equal to 2 , we can do it as follows. Let $Y \subset X$ be a lagrangian subvariety. Choose another lagrangian subvariety $Z \subset X$ such that $Y \cap Z$ is finite. Then we have a $\mathbb{G}_{m}$-valued symplectic form on $Y \cap Z$ defined by the canonical duality between $Y \cap Z=\operatorname{ker}(Y \rightarrow \hat{Z})$ and $\operatorname{ker}(Z \rightarrow \hat{Y})=Y \cap Z$. Since the characteristic is different from 2 there exists a central extension $G$ of $Y \cap Z$ by $\mathbb{G}_{m}$ with such commutator form (unique up to an isomorphism), so we have the corresponding class $e_{Y} \in$ $H^{2}\left(X / Y, \mathbb{G}_{m}\right)$ which doesn't depend on the choices made (and coincides with the one defined previously using $P$ ).

Theorem 4.3. Assume that the characteristic of the base field is different from 2. Then for every pair of lagrangian subvarieties $Y$ and $Z$ the categories $\mathcal{D}^{b}\left(X / Y, e_{Y}\right)$ and $\mathcal{D}^{b}\left(X / Z, e_{Z}\right)$ are equivalent.

Proof. As before we may assume that $Y \cap Z$ is finite. Now choose a central extension $G$ of $Y \cap Z$ inducing the canonical symplectic form on it and define the functor from the category of weight- $1 G$-equivariant objects of $\mathcal{D}^{b}(Z)$ to that of weight-1 $G^{-1}$-equivariant objects of $\mathcal{D}^{b}(Y)$ by the formula

$$
\bar{R}(\mathcal{F})_{y}=\int_{Z / H} L_{z, y} \mathcal{F}_{z} d z
$$

where $H \subset Y \cap Z$ is lagrangian. As above it is easy to check that this is an equivalence.

Remarks. 1. The constructed equivalences are not canonical and they don't agree for triples of lagrangian subvarieties. The corresponding analogue of Maslov index and a partial generalization of this theory to abelian schemes will be discussed in a forthcoming paper. 
2. The following observation is due to M. Kontsevich. If $(X, L=P \otimes$ $\left.\sigma^{*} P^{-1}\right)$ is a split symplectic biextension then the monoidal categories $\operatorname{Fun}\left(\mathcal{D}^{b}(X / Y), \mathcal{D}^{b}(X / Y)\right.$ ) (here Fun denotes the category of exact functors) for all split lagrangian subvarieties $Y \subset X$ (together with line bundles $M \in \operatorname{Pic}(Y)$ such that $\left.\left.P\right|_{Y^{2}} \simeq \Lambda(M)\right)$ are canonically equivalent. Hence, there should be a natural definition of this category not depending on a choice of $Y$. These categories of functors are rather unmanagable but we can ask the same question about the categories $\mathcal{D}^{b}(X / Y \times X / Y)$ with the monoidal structure $K * L=p_{13 *}\left(p_{12}^{*} L \otimes p_{23}^{*} K\right)$ where $p_{i j}$ are the projections from $(X / Y)^{3}$. If we take $X=\hat{A} \times A, P=p_{14}^{*} \mathcal{P}$, and $Y=\hat{A} \subset \hat{A} \times A$, then $X / Y \simeq A$ and the category $D^{b}(A \times A)$ with the above monoidal structure is equivalent to the category $\mathcal{D}^{b}(X)$ with the monoidal structure given by

$$
(K * L)_{x}=\int_{x_{1}+x_{2}=x} L_{x_{1}} \otimes K_{x_{2}} \otimes P_{x_{1}, x_{2}}
$$

The required monoidal equivalence $\mathcal{D}^{b}(X)=\mathcal{D}^{b}(\hat{A} \times A) \rightarrow \mathcal{D}^{b}(A \times A)$ is given by the functor $K \mapsto \widetilde{K}$ where

$$
\widetilde{K}_{x, y}=\int_{\xi \in \hat{A}} \mathcal{P}_{\xi, y} \otimes K_{\xi, x-y}
$$

Thus, the category $\mathcal{D}^{b}(X)$ with the monoidal structure given by (4.1) provides an answer to the above question.

\section{Appendix. Descent for derived categories}

An unpleasant property of the derived category of coherent sheaves of $\mathcal{O}_{S}$-modules on a scheme $S$ is that one can not glue this category from its counterparts over open parts of $S$. However, the following descent result holds.

Theorem A. Let $p: S^{\prime} \rightarrow S$ be a finite flat morphism. Then the category $\mathcal{D}^{b}(S)$ is equivalent to the the following category $\mathcal{D}^{b}\left(S^{\prime}, p\right)$ : its objects are pairs $(\mathcal{F}, f)$ where $\mathcal{F} \in \mathcal{D}^{b}\left(S^{\prime}\right), f: p_{1}^{*} \mathcal{F} \rightarrow p_{2}^{*} \mathcal{F}$ is an isomorphism in $\mathcal{D}^{b}\left(S^{\prime} \times{ }_{S} S^{\prime}\right)$ (where $p_{i}: S^{\prime} \times{ }_{S} S^{\prime} \rightarrow S^{\prime}, i=1,2$ are the projections) satisfying the following cocycle condition $p_{23}^{*} f \circ p_{12}^{*} f=p_{13}^{*} f$ over $S^{\prime} \times{ }_{S} S^{\prime} \times{ }_{S} S^{\prime}$.

Proof. Let $p^{*}: \mathcal{D}^{b}(S) \rightarrow \mathcal{D}^{b}\left(S^{\prime}, p\right)$ be the natural functor. Let us check first that $p^{*}$ is fully faithful. Assume that we have a morphism $f: p^{*} \mathcal{F} \rightarrow p^{*} \mathcal{G}$ in $\mathcal{D}^{b}\left(S^{\prime}\right)$ such that the following diagram is commutative: 


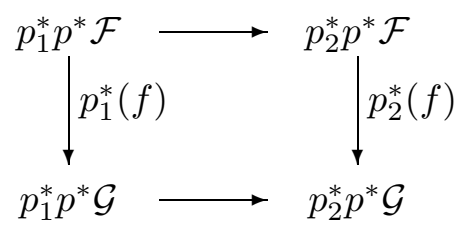

Applying the functor $p_{1 *}$ to this diagram and composing it with the adjunction morphism $p^{*} \mathcal{F} \rightarrow p_{1 *} p_{1}^{*} p^{*} \mathcal{F}$ we get the following diagram

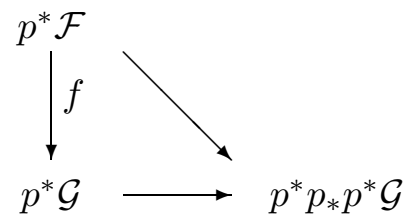

where the diagonal morphism is $p^{*} f^{\prime}, f^{\prime}: \mathcal{F} \rightarrow p_{*} p^{*} \mathcal{G}$ is obtained from $f$ by adjunction. Let us denote by $f^{\prime \prime}$ the composition

$$
\mathcal{F} \stackrel{f^{\prime}}{\rightarrow} p_{*} p^{*} \mathcal{G} \simeq p_{*} \mathcal{O}_{S^{\prime}} \otimes \mathcal{G} \rightarrow\left(p_{*} \mathcal{O}_{S^{\prime}} / \mathcal{O}_{S}\right) \otimes \mathcal{G} .
$$

Then it follows from the diagram above that $p^{*}\left(f^{\prime \prime}\right)=0$. Since $p_{*} p^{*}(?) \simeq$ $p_{*} \mathcal{O}_{S^{\prime}} \otimes$ ? it follows that $f^{\prime \prime}=0$, hence $f^{\prime}$ factors through a morphism $\bar{f}: \mathcal{F} \rightarrow \mathcal{G}$ and $f=p^{*}(\bar{f})$. Thus, the functor $p^{*}: \mathcal{D}^{b}(S) \rightarrow \mathcal{D}^{b}\left(S^{\prime}, p\right)$ is full and faithful. It remains to check that any object of $\mathcal{D}^{b}\left(S^{\prime}, p\right)$ belongs to its essential image. This is easy to prove by devissage with respect to the standard $t$-structure on $\mathcal{D}^{b}\left(S^{\prime}\right)$ since the corresponding truncation functors are compatible with descent data (the base of induction is provided by the classical descent for coherent sheaves).

\section{Acknowledgement}

I am grateful to M. Kontsevich for valuable remarks.

\section{References}

1. L. Breen, Functions theta et théorème du cube, Lecture Notes in Math., vol. 980, Springer-Verlag, 1983.

2. P. Deligne, Le symbole modéré, Publ. Math. IHES 73 (1991), 147-181.

3. L. Moret-Bailly, Pinceaux de variétés abéliennes, Astérisque 129 (1985).

4. D. Mumford, Abelian varieties. Second edition. Oxford University Press. 1974.

5. A. Polishchuk, Biextensions, Weil representation on derived categories and thetafunctions. Ph. D. Thesis, Harvard University, 1996.

Department of Mathematics, Harvard University, Cambridge, MA 02138

E-mail address: apolish@math.harvard.edu 